\title{
Decoupling the Cortical Power Spectrum Reveals Real-Time Representation of Individual Finger Movements in Humans
}

\author{
K. J. Miller, ${ }^{1}$ S. Zanos, ${ }^{2}$ E. E. Fetz, ${ }^{2}$ M. den Nijs, ${ }^{1}$ and J. G. Ojemann ${ }^{3}$ \\ Departments of ${ }^{1}$ Physics and ${ }^{2}$ Physiology and Biophysics, and ${ }^{3}$ Center for Neuroscience, Seattle Children's Research Institute and Department of \\ Neurological Surgery, University of Washington, Seattle, Washington 98195
}

During active movement the electric potentials measured from the surface of the motor cortex exhibit consistent modulation, revealing two distinguishable processes in the power spectrum. At frequencies $<40 \mathrm{~Hz}$, narrow-band power decreases occur with movement over widely distributed cortical areas, while at higher frequencies there are spatially more focal power increases. These high-frequency changes have commonly been assumed to reflect synchronous rhythms, analogous to lower-frequency phenomena, but it has recently been proposed that they reflect a broad-band spectral change across the entire spectrum, which could be obscured by synchronous rhythms at low frequencies. In 10 human subjects performing a finger movement task, we demonstrate that a principal component type of decomposition can naively separate low-frequency narrow-band rhythms from an asynchronous, broad-spectral, change at all frequencies between 5 and $200 \mathrm{~Hz}$. This broad-spectral change exhibited spatially discrete representation for individual fingers and reproduced the temporal movement trajectories of different individual fingers.

\section{Introduction}

Recording neural activity in the brain with high spatiotemporal resolution is crucial for investigating brain function and controlling brain-machine interfaces. Such resolution has been sought by measuring the activity of single or multiple neurons using microelectrodes implanted inside the brain. A less invasive and clinically more applicable measure, electrocorticography (ECoG), recorded from the surface of the brain, measures the behaviorally modulated (Penfield and Boldrey, 1937; Gastaut, 1952) aggregate activity of cortical neural populations, with high temporal precision. During movements, low frequencies of the ECoG signal [the $\alpha(8-12 \mathrm{~Hz})$ and $\beta(18-25 \mathrm{~Hz})$ rhythms] show a characteristic decrease in power in over large areas of sensorimotor cortex (Arroyo et al., 1993; Crone et al., 1998b; Aoki et al., 1999). Movements are also associated with an increase in power in higher frequencies $(>75 \mathrm{~Hz})$, with a more focal cortical distribution (Crone et al., 1998a; Aoki et al., 1999; Miller et al., 2007b; Miller et al., 2007d).

Both low and high frequency task-related changes have been attributed to decreased or increased synchrony between the neural populations that give rise to these changes (Crone et al., 1998a; Aoki et al., 1999; Pfurtscheller et al., 2003; Canolty et al., 2006). However, in all of these studies, specific frequency ranges of the power spectral density (PSD) were selected for analysis and the results and their interpretation were tied to frequency-specific

Received Nov. 14, 2008; revised Jan. 14, 2009; accepted Feb. 9, 2009.

This work was supported by the National Science Foundation (Grants 0622252 and 0130705). We thank R. Rao, L. Sorensen, S. Makeig, G. Schalk, J. Onton, D. Hermes, B. Pesaran, and A. Fairhall for constructive discussion, and the patients and staff at Harborview Hospital in Seattle, WA for their participation.

Correspondence should be addressed to Kai J. Miller, Department of Physics, Box 351560, University of Washington, Seattle, WA 98195. E-mail: kjmiller@u.washington.edu.

D01:10.1523/JNEUROSCI.5506-08.2009

Copyright $\odot 2009$ Society for Neuroscience $\quad$ 0270-6474/09/293132-06\$15.00/0 processes, a practice which, by itself, implies neuronal synchrony at specific timescales.

We report a new method of extracting and removing the low frequency $\alpha$ and $\beta$ rhythms to reveal behaviorally modulated broadband changes in the ECoG signal, across all frequencies. The amplitude of this broadband change provides significantly improved spatial resolution of local cortical populations, and is dynamically modulated with high temporal resolution $(<20 \mathrm{~ms})$. This extraction technique has provided the first resolution of real-time signals correlated with individual finger movements in neighboring sites of human motor cortex.

We hypothesized (Miller et al., 2007d), and later demonstrated (Miller et al., 2007a), that the high-frequency power increase is part of a broad-band phenomenon of the form $P \sim A f^{-\chi}$ (where $P=$ power; $f=$ frequency; $A$ and $\chi$ are constants). In this study we show that this broad-band power law pertains across the entire power spectrum, although it is obscured by changes in narrow-band oscillations at lower frequencies. We further show that this broadband phenomenon is spatially localized and specific to the function of the underlying cortical area. To explore whether this broadband phenomenon existed, and could be naively extracted from the narrow band oscillations, we investigated a set of tasks designed to engage cortical areas that were sufficiently close together to exhibit similar changes in low-frequency rhythms for the different tasks, yet sufficiently separated to avoid overlap of any task-specific broadband, spatially focal, phenomenon. The different covariance in the spectral density of these two processes across tasks would allow decomposition by principal-component analysis. Based upon preliminary findings (Miller et al., 2007d), we hypothesized that individual fingers are represented at separate cortical sites, and thus a simple isolated finger movement task would meet these criteria. 


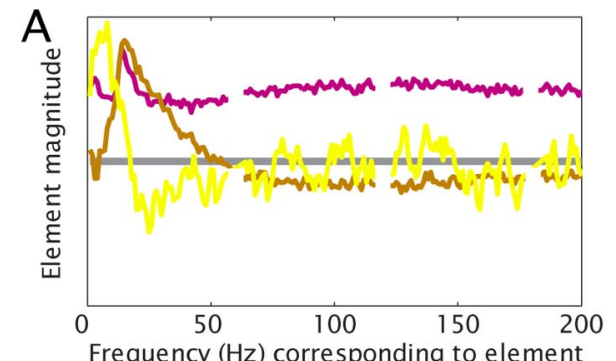

Frequency $(\mathrm{Hz})$ corresponding to element
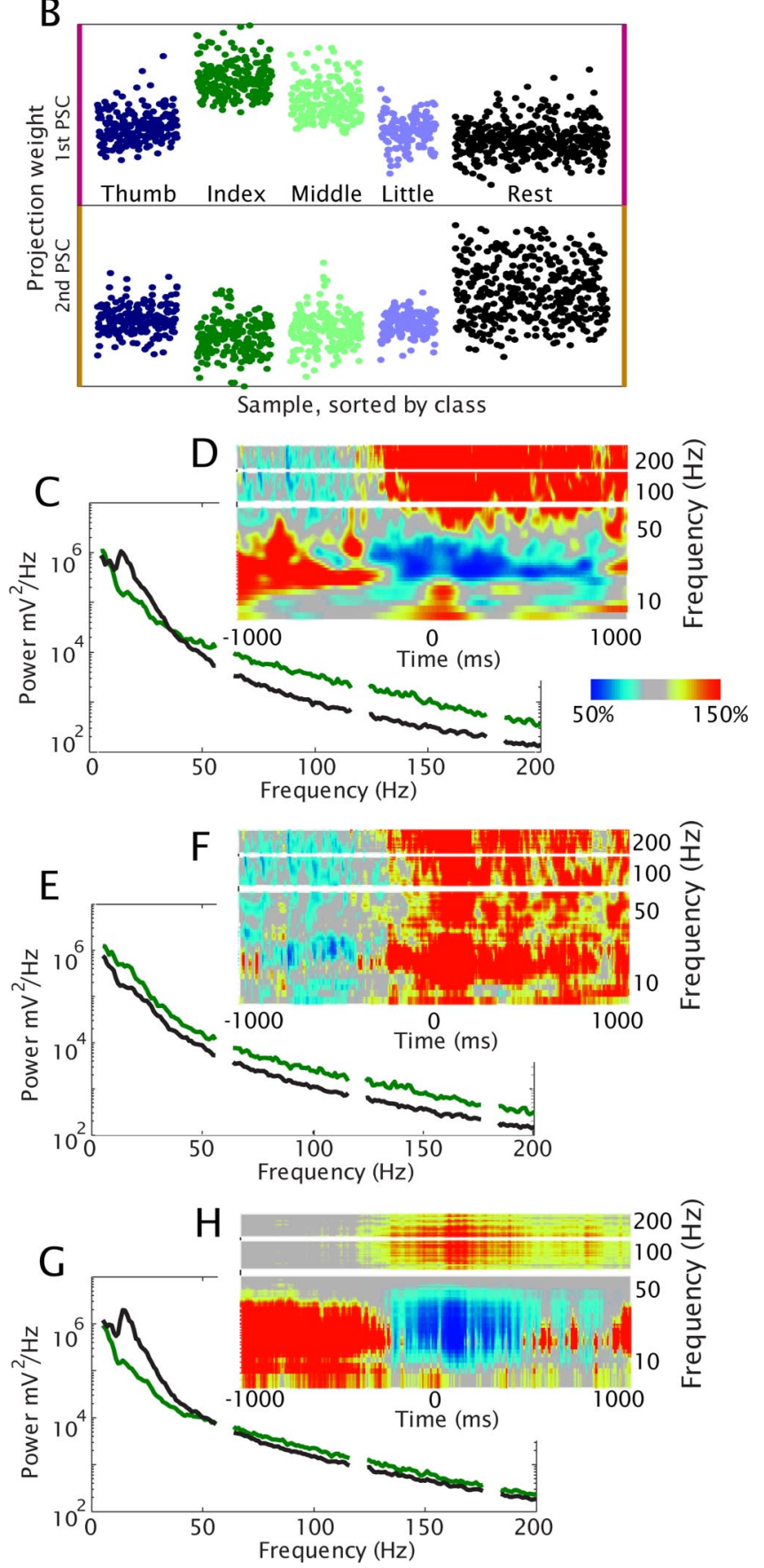

Figure 1. Representative spectral changes in a single electrode from motor cortex. In response to visual cues, subject 6 repeatedly flexed and extended different fingers of his contralateral hand. Samples of the normalized PSD of the potential timeseries were calculated from $1 \mathrm{~s}$ windows centered at times of maximum flexion and also during rest. $\boldsymbol{A}$, Normalized PSD samples were naively decomposed using a principal component approach to characterize covariation in power at different frequencies. The elements of the first principal spectral component (first PSC, pink) are non-zero across all frequencies, consistent with change in a power-law in

\section{Materials and Methods}

Subjects and recordings. Ten epileptic patients at Harborview Hospital in Seattle, WA, volunteered, and provided informed consent, to participate in the study (18- to 45-year-old, 6 female). Each had sub-dural electrocorticographic (ECoG) grids placed for extended clinical monitoring and localization of seizure foci. Stimulation mapping was performed clinically, and anonymized recording and anatomical data were provided to researchers.

Experiments were performed at the patients' bedside, and the experimental signal was amplified in parallel with clinical recording. The electrode arrays consisted of 32-64 circular platinum contacts $(2.3 \mathrm{~mm}$ exposed diameter) embedded in a SILASTIC sheet, and surgically placed on the sub-dural brain surface. Electrode positions were calculated using $\mathrm{x}$-rays and plotted to the AFNI standardized template brains using the LOC package (Miller et al., 2007c). Cortical potentials were sampled at $1000 \mathrm{~Hz}$, with respect to a scalp reference and ground. Movement cues were presented with a monitor at the bedside using the general-purpose BCI2000 stimulus and acquisition program (Schalk et al., 2004), which also recorded the behavioral parameters and cortical data. In five of the patients, cortical stimulation mapping (Ojemann et al., 1989) of motor cortex was performed for clinical purposes.

Movement task. Subjects were cued with a word displayed on a bedside monitor to move fingers independently during $2 \mathrm{~s}$ movement trials. They typically moved a finger 3-5 times during each trial, but some trials included many more movements. A 2 s rest trial (blank screen) followed each movement trial. There were 30 movement cues for each finger, and trial types were interleaved randomly (typically 100-150 total movements per finger). Finger position was recorded using a 5 degree-offreedom dataglove (see Fig. 2). Event markers were calculated marking the initiation of movement following a cue, and the time of peak flexion from each finger movement (times of peaks denoted by $\tau_{q}$ ). "Rest" events, which were included in $\tau_{q}$ were defined during random periods occurring at least $500 \mathrm{~ms}$ from any movement initiation or termination, and separated by at least $250 \mathrm{~ms}$ from any other rest event (there were typically 150-250 such rest events). A $37 \mathrm{~ms}$ ( $\pm 3 \mathrm{~ms}$, SEM) lag occurred between the Dataglove position measurement recording and the amplifier measurement and was accounted for when calculating the latency between brain activity and finger movement.

Discrete spectral analysis and decomposition. The scalp-referenced ECoG potentials were re-referenced with respect to the common average reference across all $N$ electrodes. From each electrode, samples of power spectral density [PSD; $P\left(f, \tau_{q}\right)$ ] were calculated from $1 \mathrm{~s}$ epochs centered at the time of maximum flexion during each movement, $\tau_{q}$. Individual $P\left(f, \tau_{q}\right)$, were normalized in two steps: each spectral sample was elementwise divided by the average across the ensemble, at each frequency, and then the log was taken.

$$
\tilde{P}\left(f, \tau_{q}\right)=\ln \left(P\left(f, \tau_{q}\right)\right)-\ln \left(\frac{1}{N_{q}} \sum_{p=1}^{N_{q}} P\left(f, \tau_{p}\right)\right) .
$$

$\leftarrow$

the cortical power spectral density. The second PSC (gold) is peaked between 15 and $25 \mathrm{~Hz}$ ( $\beta$ rhythm range). The third PSC (yellow) is a $0-10 \mathrm{~Hz}(\theta, \alpha)$ peak. (4th -179 th PSCs not shown). $B$, Projection magnitudes to each spectral sample from the first (top) and second (bottom) PSCs, sorted by movement type; black indicates samples from rest periods. Therefore, each such sample, denoted by a dot, corresponds to the contribution of a PSC to the PSD from a one second epoch flanking a single movement, and there are several such movements in response to a single cue. Note that the first PSC has a specific increase from rest for index finger movements, and, to a lesser degree, for middle finger. The second PSC shows decrease from rest for all finger types. C, Mean PSD of index finger movement samples (dark green) and rest samples (black). $\boldsymbol{D}$, Average time-varying PSD (scaled as percentage of mean power at each frequency) with respect to first index finger movement from each movement cue $(N=30)$. $\boldsymbol{E}$, Mean of reconstructed PSD samples from the same electrode, and $(\boldsymbol{F})$ average reconstructed time-varying PSD, with second and third PSC omitted. This is consistent with the increase in the amplitude of a power law with local cortical activity. $\boldsymbol{G}$, Mean of reconstructed PSD samples, and $(\boldsymbol{H})$ average reconstructed time-varying PSD, using only second and third PSCs. This is consistent with a change in the $\alpha$ and $\beta$ rhythms. Note that $\boldsymbol{C}=\boldsymbol{E}+\boldsymbol{G}$ and $\boldsymbol{D}=\boldsymbol{F}+\boldsymbol{H}$. These findings generalize across all subjects and electrodes (supplemental Fig $S 4$, available at www.jneurosci.org as supplemental material). 
The label $q$ refers to times of peak movement for all finger movement types, and times of rest where no movement was taking place (total number of movement and rest events $\tau_{q}$ denoted $N_{q}$ ). A principal component method (Jolliffe, 2002) was then applied to try to identify motifs of movement-related change in the PSD. In the first stop of this decomposition, the covariance matrix $C(f, \tilde{f})$ between frequencies is calculated: $C(f, \tilde{f})=\sum_{\tau_{q}} \tilde{P}\left(f, \tau_{q}\right) \tilde{P}\left(\tilde{f}, \tau_{q}\right)$. The eigenvalues, $\lambda_{k}$, and eigenvectors, $\vec{e}_{k}$, of this matrix reveal the robust common features during movement. We name these $\vec{e}_{k}$ "Principal Spectral Components" (PSCs), and order them according to importance, as determined by the magnitude of the corresponding $\lambda_{k}$. The original power spectral density, for particular movement and rest classes, was reconstituted without one or more of the PSCs to illustrate motifs in the movement-associated spectral change. Three electrodes from each subject were selected for display and further analysis, based upon the maximum cross-correlation between projection weight samples from thumb, index, or little finger movement, and samples of rest (as illustrated on the axes in Fig. 2 B).

Continuous spectral analysis and decomposition. Continuous timefrequency approximations (dynamic spectra) were calculated using a wavelet approach. In each channel, the time-varying continuous Fourier component was calculated, with fixed uncertainty between the estimate of the instantaneous amplitude and phase versus the temporal resolution, using a complex Morlet filter at each Hz. Movement-onset averaged PSD change (normalized by division at each $\mathrm{Hz}$ ) could be examined and decomposed (after taking the log, to obtain a fully normalized $\tilde{P}(f, t)$ ) using the same procedure as the discrete case. The projection of each principal spectrum could then be estimated at each point in time, by back-projecting the continuous spectra, $e_{k}(f) * \tilde{P}(f, t)$, smoothing, normalizing, and re-exponenting to obtain the quantity $C_{k}(t)$ (denoting the time course of the $k$ th PSC, not the covariance matrix $C(f, \tilde{f}))$.

\section{Results}

\section{Movement-related spectral changes}

Analysis of the ECoG potentials recorded during the task reveals that just before the initiation of finger movement, there is the characteristic decrease in power at lower frequencies $(\alpha / \beta$ range), and a spectrally broad, but spatially focal, increase in power at higher frequencies of the ECoG PSD (above $\sim 40 \mathrm{~Hz}$ ) (Figs. $1 E$, $2 D$; supplemental Fig. $S 4 E$, available at www.jneurosci.org as supplemental material). This is not due to an event-related potential (supplemental Fig. S6, available at www.jneurosci.org as supplemental material) and has also been observed for coarser movements (Crone et al., 1998a; Aoki et al., 1999; Miller et al., 2007b; Miller et al., 2007d). A principal component decomposition of these changes revealed robust, characteristic, motifs (Fig. 1) which were consistent and reproducible across all subjects and electrodes in hand motor cortex during finger movement (supplemental Fig. S4, available at www.jneurosci.org as supplemental material). We found that the largest and most significant motif in PSD change, i.e., the most significant principal component (first PSC), consistently showed, across all subjects and electrodes, the frequency element magnitudes that were non-zero, with the same sign, where each element was closer to the average across all elements than it was to zero (pink traces in Figs. $1 A$, $2 C-E$, supplemental Fig. S4, available at www.jneurosci.org as supplemental material). This suggests that the reported "high $\gamma$ " changes (Brovelli et al., 2005; Canolty et al., 2006) may, in fact, be a reflection of this broad spectral phenomenon (supplemental Fig. S15, available at www.jneurosci.org as supplemental material). The second PSC was peaked in the $\alpha / \beta$ range, reflecting a process in a narrow range of frequencies, beginning near zero and peaking at a low frequency (Fig. $1 A$; supplemental Fig. S4A, available at www.jneurosci.org as supplemental material). This finding is consistent with the phenomenon of "event-related desynchronization" (ERD) (Pfurtscheller, 1999), and likely reflects resting rhythms that decrease when the cortical areas are acti- vated. The methodological constraints in the process were first that the decoupling was less robust in the cases where behavioral variance was not robust (fingers were not moved independently), and second that the partially shared variance between the broad spectral phenomenon and the ERD resulted in the presence of residual amounts of each both of the first two PSCs.

\section{The spatial distribution of the principal spectral components on the cortical surface}

The relative strength of the first PSC was different at neighboring cortical locations, revealing separable individual finger somatotopy in all subjects (Figs. 2, 3; supplemental Fig. S5, available at www.jneurosci.org as supplemental material). While there was some variability in somatotopic organization, the most common organization showed thumb, index, middle, and little fingers represented in ventral to dorsal order. (We excluded the ring finger because its movements were highly correlated with movements of the middle or little finger in every subject, as shown in supplemental Fig. S13, available at www.jneurosci.org as supplemental material.) We found mostly distinct representations of different fingers; furthermore, much of the apparent overlap in cortical representation in digits was associated with the well-known correlation in the movement of the fingers (Schieber, 1995) (supplemental Fig. S13, available at www.jneurosci.org as supplemental material). Although the approximated positions of the most significant electrodes did not always lie on the precentral gyrus of the template cortex, the motor somatotopy identified by the first PSC consistently agreed with the sites that produced finger motor movement when stimulated with electrical current [supplemental Fig. S11, available at www.jneurosci.org as supplemental material, performed for clinical purposes in the same way as the classic electrocortical stimulation studies (Penfield and Boldrey, 1937; Woolsey et al., 1952, 1979)].

For projection to the second PSC, all finger movement types were different from rest at each of the three sites, and corresponded to decreases in power in the $\alpha / \beta$ range (Fig. 2C-E; supplemental Fig. S5, available at www.jneurosci.org as supplemental material). This corresponded to a much more broad spatial distribution of change (Miller et al., 2007d). The gradient observed between movement types in this second PSC is suggestive of the overlapping gradient representation described by recent imaging and lesion studies (Kleinschmidt et al., 1997; Schieber, 1999; Kim, 2001; Dechent and Frahm, 2003).

\section{The correlation between the time course of the first PSC and finger movement dynamics}

The time course of the first PSC, $C_{1}(t)$ measured from individual electrodes was highly correlated with finger position, and was specific for individual digits, with average correlation of $r=0.37$, and as high as $r=0.62$, over $10 \mathrm{~min}$ of continuous recording (Figs. 2, 4). The mean latency in the correlation peak of $\sim 85 \mathrm{~ms}$ (Fig. 4) is consistent with the delay between cortical signals and movement initiation (Evarts, 1973; Cheney and Fetz, 1984). This high correlation, specific for movement of a single finger, demonstrates that activation of local neural populations can be measured at a temporal resolution of tens of milliseconds, and a spatial resolution of at least $0.5-1 \mathrm{~cm}$, the spacing of the clinical ECoG array.

\section{Discussion}

Single-unit recordings in behaving monkeys have shown that M1 neurons correlated with movement of different fingers are broadly distributed and intermingled (Schieber and Hibbard, 

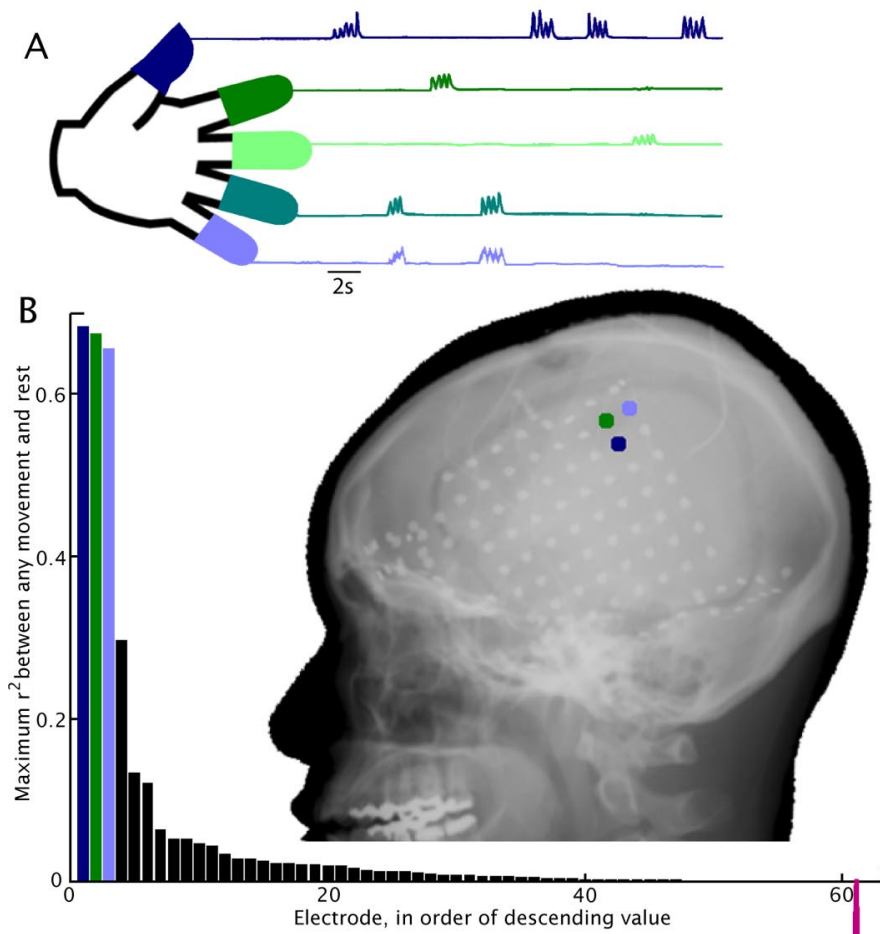

D
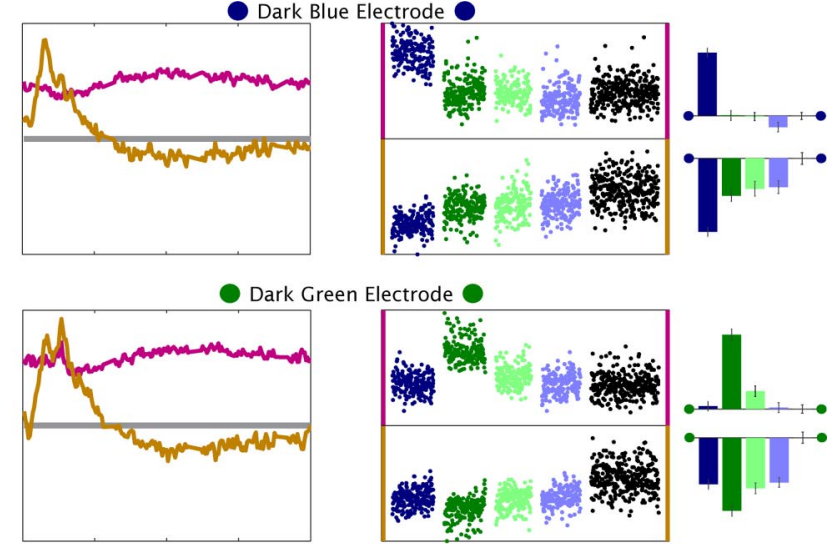

Dark Green Electrode

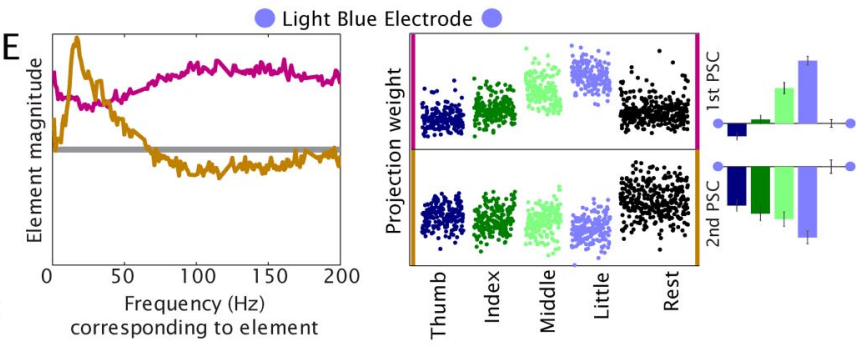

F Thumb Position

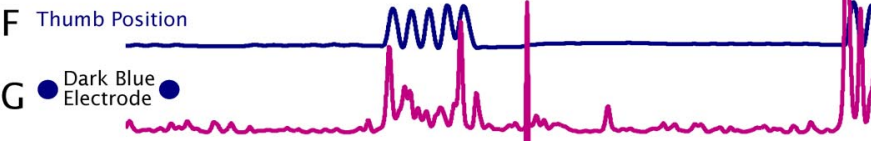

M
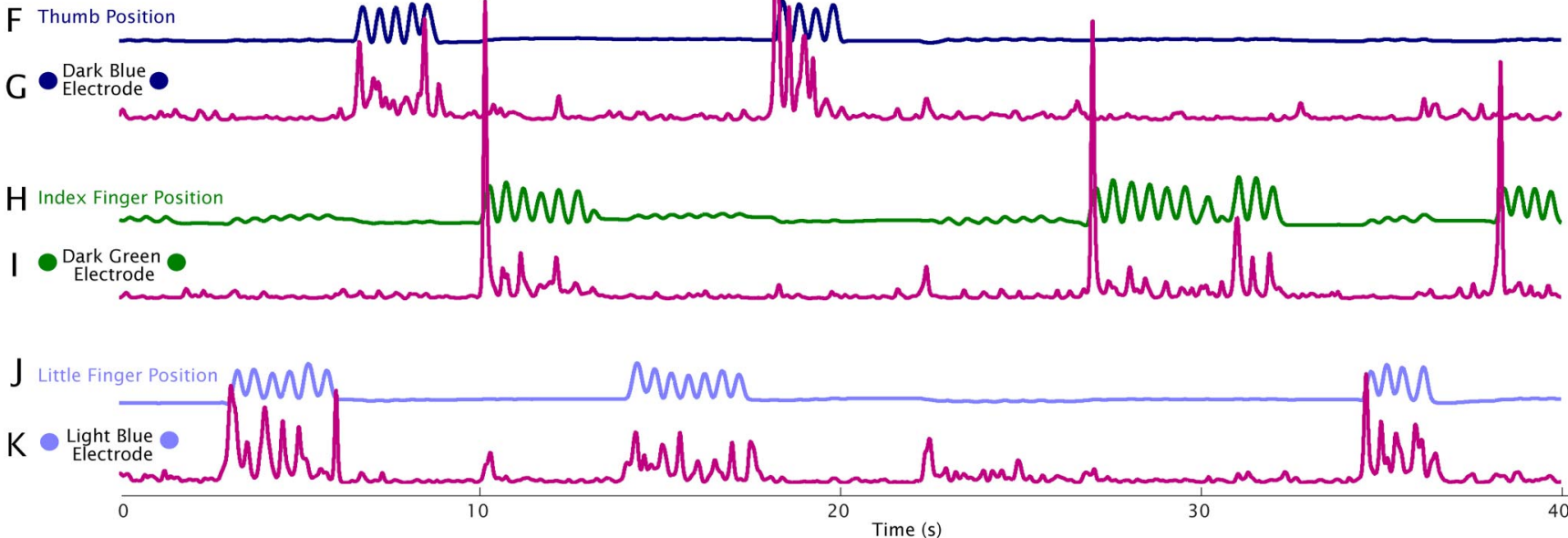

Figure 2. Capturing local cortical activity; individual digit representation in adjacent electrodes in subject 1. $\boldsymbol{A}$, The position of each finger was measured using a transducing glove during cued flexion-extension. $\boldsymbol{B}$, Axes, The maximum squared cross-correlation $\left(r^{2}\right)$ between sample projection weights to the first PSC, for each electrode, between any single movement type and rest, illustrates sparse digit representation (supplemental Figs. $54-6$, available at www.jneurosci.org as supplemental material). Corresponding locations are shown in $\mathrm{x}$-ray. Each of these shows specificity for a different type of finger movement, and the color code redundancy between the electrodes in $\boldsymbol{B}-\boldsymbol{K}$ and digit movement type in $\boldsymbol{A}$ reflects this. $\boldsymbol{C}$, Left, First (pink) and second (gold) PSCs generated from finger movements and rest, for the electrode shown in dark blue in the $x$-ray inset in $B$. Middle, As in Figure $1 B$. Right, Mean projection magnitudes for each finger type, with mean from rest samples subtracted. Error bars indicate \pm 3 times the $S E(3 \sigma)$ of the mean (right most: rest samples). The upper bars represent the first PSC, and the lower are for the second. $\boldsymbol{D}, \boldsymbol{E}$, As in $\boldsymbol{C}$, but for the dark green $(\boldsymbol{D})$ and light blue $(\boldsymbol{E})$ electrodes. $\boldsymbol{F}, \boldsymbol{H}, \boldsymbol{J}$, Traces of thumb, index, and little finger position for a $40 \mathrm{~s}$ period. $\boldsymbol{G}, \boldsymbol{I}, \boldsymbol{K}$, Projections of the time-frequency representation to the first PSC, " $C_{1}(t)$ ", for each of the three electrodes for same $40 \mathrm{~s}$. Each electrode is specifically and strongly correlated with one movement type $\left(r=0.46 C_{1}(t)\right.$ from dark blue electrode-thumb; $r=$ 0.47 green electrode-index finger; $r=0.29$ light blue electrode-little finger; cross-combinations had a mean correlation of -0.09 , indicating light hyperextension of other fingers while flexing the appropriate finger in this subject), over $10 \mathrm{~min}$ of continuous data $\left(3.6 \times 10^{6}\right.$ samples).

1993; Schieber, 2001). Axon tracing studies have found widespread projections of single descending M1 axons to multiple motor neuronal pools across different joints (Shinoda et al., 1979). Physiological studies show convergence of axons from widespread territories in M1 onto single motor neurons of hand muscles (Phillips and Porter, 1977) and divergence of effects from single M1 cells to multiple muscles (Cheney, 1980; Porter and Lemon, 1993) Our findings suggest that this highly overlapping representation at the single neuron level can, at a coarser spatial scale, be resolved by an aggregate signal generated by the local neuronal population at large.

We believe that this integrated population signal provides information that is not captured by intracortical recordings through high-impedance microelectrodes. High frequencies in the unit recordings are dominated by single or multiple individual unit activity from nearby neurons, which individually may not be representative of event-related changes in the overall local neuronal population. In contrast, the cortical surface electrodes, 

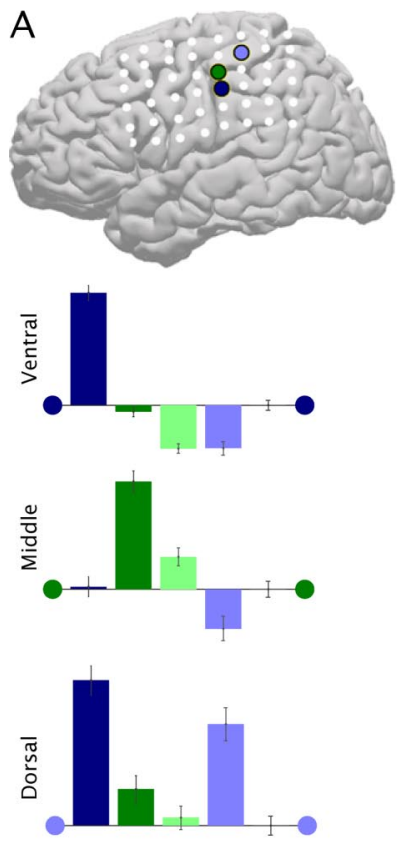
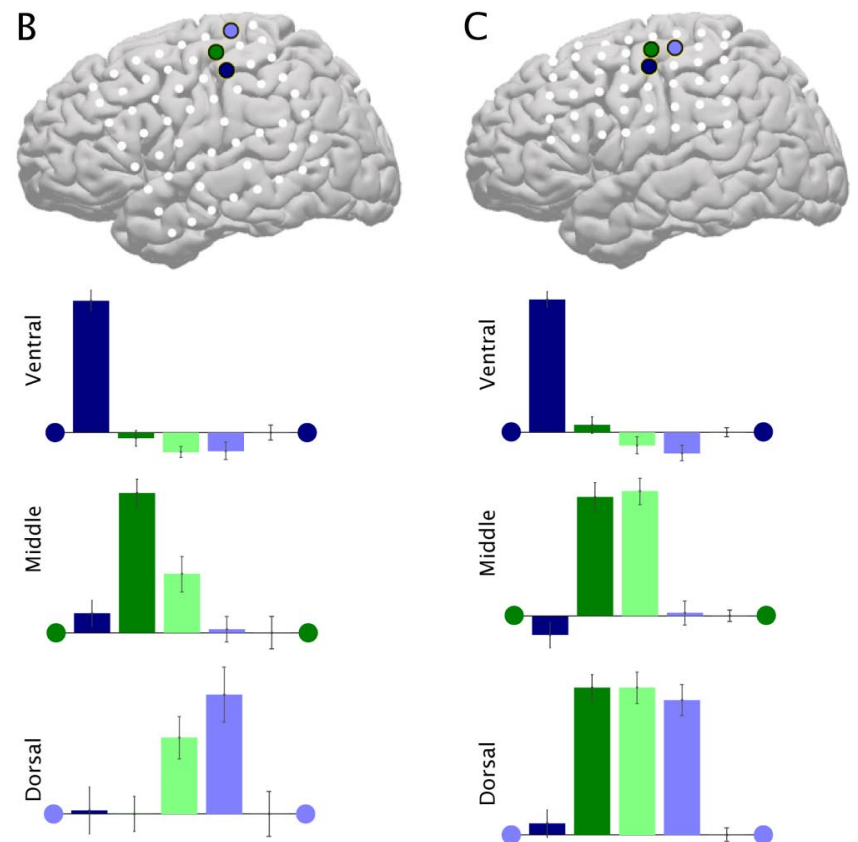
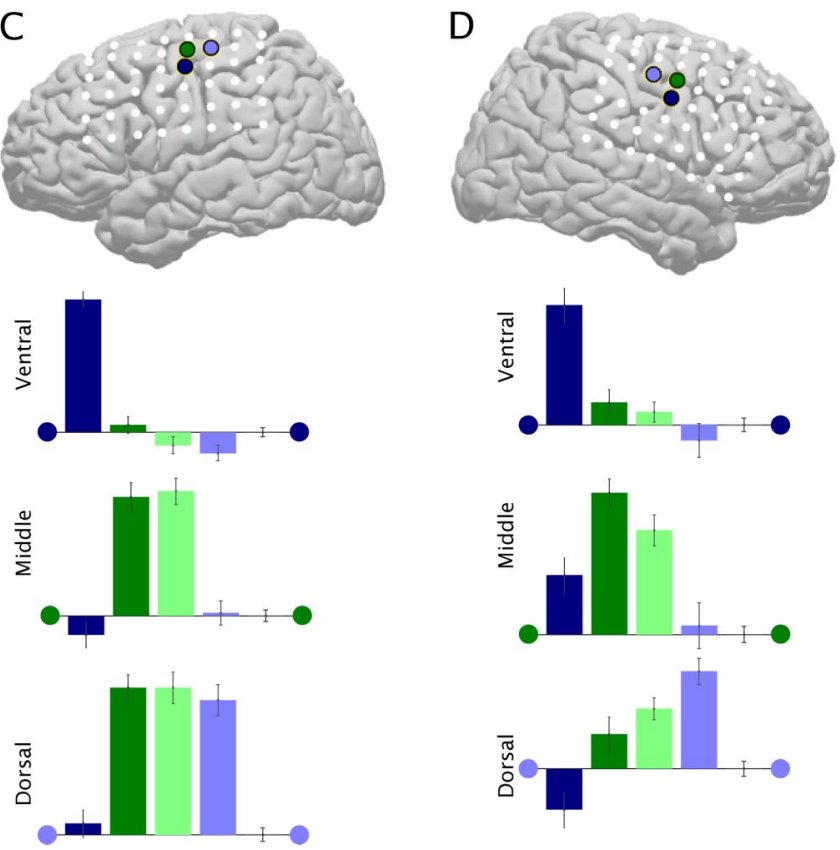
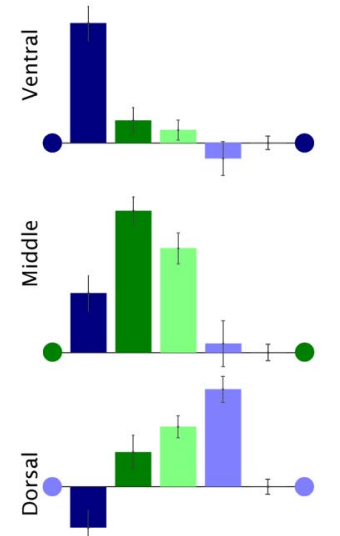

Figure 3. First PSC projection magnitudes, at three surface electrodes. $\boldsymbol{A}$, Mean of projection magnitudes in three electrodes of samples for each finger movement class, after subtraction of the mean of the rest time samples, for subject 2 (as in the right-hand column of insets $\boldsymbol{C} \boldsymbol{E}$ in Fig. 2). The color of the dots flanking axes indicate corresponding electrode on the cortical schematic. Redundancy in color between electrode color and movement type is meant to provide intuitive relation, but also indicates electrode-movement type pairings used for analysis. $\boldsymbol{B}-\boldsymbol{D}$, Same as $\boldsymbol{A}$, but for subjects 3-5 (B, subject 3; $\boldsymbol{C}$, subject 4; $\boldsymbol{D}$, subject 5).

with their large contact areas, pick up and "average" aggregate activity from large neuronal populations. These neuronal ensembles can be partially synchronized over large distances at specific, low-frequency, timescales; this is manifested as frequencyspecific event-related desynchronization, and is evident in both intracortical (Baker et al., 1999) and epicortical (Crone et al., 1998a; Miller et al., 2007d) recordings. We find that the spatial distribution of this synchronous process does not exhibit strong specificity for individual finger movements. On the other hand, the broadband signal we extracted at certain cortical sites is specific for movement of individual fingers, in agreement with the more focal spatial distribution of highfrequency components (Crone et al., 1998a; Miller et al., 2007d), which are themselves part of this broadband process (supplemental Fig. S15, available at www. jneurosci.org as supplemental material). What could be the physiological source of such a broadband signal?

We have previously found empirically that the integrated signal is described by a power law, $P \sim A f^{-\chi}$, which changes in amplitude, but not form, with activity (Miller et al., 2007a), as captured by the first PSC. Such a power law distribution is consistent with a coarse-grained measurement of local, Poisson-distributed, synaptic inputs to the $\sim 5 \times 10^{5}$ neurons in the cortical region beneath each electrode, without a favored timescale (Brovelli et al., 2005; Canolty et al., 2006). We suggest that, although $(\mathrm{SD}=39 \mathrm{~ms}, \mathrm{~N}=24)$.
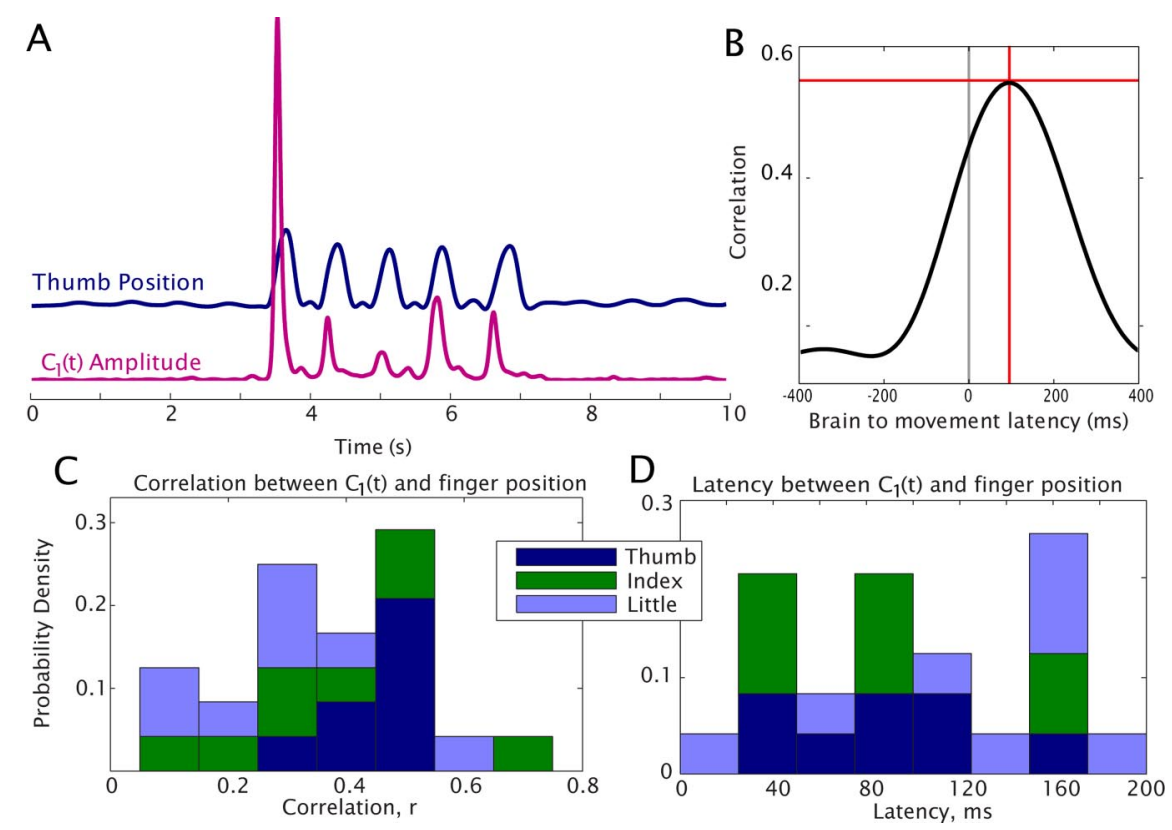

Figure 4. Correlation between the time course of the first $\operatorname{PSC}\left(C_{1}(t)\right)$ and finger position. $A, A$ single trial showing $C_{1}(t)$ in an associated electrode and thumb movement, demonstrating delay from the cortex to movement onset, from subject $10 . \boldsymbol{B}$, Correlation in the electrode-movement pair from $A$ as a function of latency between the two, over the full 10 min period. $C$, The correlation between $C_{1}(t)$ and paired finger movement was $0.37(S D=0.16, N=24)$ across eight of the subjects, three electrodes each (subjects 2 and 8 excluded, because their movement-associated cortical change was poorly separated; supplemental Fig. S7, available at www.jneurosci.org as supplemental material). $\boldsymbol{D}$, The mean latency between $\boldsymbol{C}_{1}(t)$ and finger movement was $84 \mathrm{~ms}$

these neuronal populations are partially synchronized to a low-frequency, $\beta(10-30 \mathrm{~Hz})$ oscillation (Murthy and Fetz, 1996), the aggregate broadband, asynchronous, activity independently maintains specificity for different classes of finger movement. 
This finding of a separable, continuous, measure for different finger movements in adjacent cortical areas supports further use of digit-based paradigms to study human motor cortex, and for use in applications such as clinical mapping and brain-machine interfaces (Acharya et al., 2008). The isolation of this broad-band, power-law, phenomenon in the cortical spectrum promises to be a powerful new method of accessing the activity of local cortical populations, resolved at behavioral timescales, in a wide range of functional contexts.

\section{References}

Acharya S, Tenore F, Aggarwal V, Etienne-Cummings R, Schieber MH, Thakor NV (2008) Decoding individuated finger movements using volumeconstrained neuronal ensembles in the M1 hand area. IEEE Trans Neural Syst Rehabil Eng 16:15-23.

Aoki F, Fetz EE, Shupe L, Lettich E, Ojemann GA (1999) Increased gammarange activity in human sensorimotor cortex during performance of visuomotor tasks. Clin Neurophysiol 110:524-537.

Arroyo S, Lesser RP, Gordon B, Uematsu S, Jackson D, Webber R (1993) Functional significance of the mu rhythm of human cortex: an electrophysiologic study with subdural electrodes. Electroencephalogr Clin Neurophysiol 87:76-87.

Baker SN, Kilner JM, Pinches EM, Lemon RN (1999) The role of synchrony and oscillations in the motor output. Exp Brain Res 128:109-117.

Brovelli A, Lachaux JP, Kahane P, Boussaoud D (2005) High gamma frequency oscillatory activity dissociates attention from intention in the human premotor cortex. Neuroimage 28:154-164.

Canolty RT, Edwards E, Dalal SS, Soltani M, Nagarajan SS, Kirsch HE, Berger MS, Barbaro NM, Knight RT (2006) High gamma power is phaselocked to theta oscillations in human neocortex. Science 313:1626-1628.

Cheney PD (1980) Response of rubromotoneuronal cells identified by spike-triggered averaging of EMG activity in awake monkeys. Neurosci Lett 17:137-142.

Cheney PD, Fetz EE (1984) Corticomotoneuronal cells contribute to longlatency stretch reflexes in the rhesus monkey. J Physiol 349:249-272.

Crone NE, Miglioretti DL, Gordon B, Lesser RP (1998a) Functional mapping of human sensorimotor cortex with electrocorticographic spectral analysis. II. Event-related synchronization in the gamma band. Brain 121:2301-2315.

Crone NE, Miglioretti DL, Gordon B, Sieracki JM, Wilson MT, Uematsu S, Lesser RP (1998b) Functional mapping of human sensorimotor cortex with electrocorticographic spectral analysis. I. Alpha and beta eventrelated desynchronization. Brain 121:2271-2299.

Dechent P, Frahm J (2003) Functional somatotopy of finger representations in human primary motor cortex. Hum Brain Mapp 18:272-283.

Evarts EV (1973) Motor cortex reflexes associated with learned movement. Science 179:501-503.

Gastaut H (1952) Electrocorticographic study of the reactivity of rolandic rhythm (in French). Rev Neurol 87:176-182.

Jolliffe I (2002) Springer series in statistics. Principal component analysis. New York: Springer.

Kim JS (2001) Predominant involvement of a particular group of fingers due to small, cortical infarction. Neurology 56:1677-1682.

Kleinschmidt A, Nitschke MF, Frahm J (1997) Somatotopy in the human motor cortex hand area. A high-resolution functional MRI study. Eur J Neurosci 9:2178-2186.

Miller KJ, Sorensen LB, Ojemann JG, den Nijs M (2007a) ECoG observations of power-law scaling in the human cortex. Arxiv preprint arXiv:0712.0846.

Miller KJ, denNijs M, Shenoy P, Miller JW, Rao RP, Ojemann JG (2007b) Real-time functional brain mapping using electrocorticography. Neuroimage 37:504-507.

Miller KJ, Makeig S, Hebb AO, Rao RP, denNijs M, Ojemann JG (2007c) Cortical electrode localization from X-rays and simple mapping for electrocorticographic research: The "Location on Cortex" (LOC) package for MATLAB. J Neurosci Methods 162:303-308.

Miller KJ, Leuthardt EC, Schalk G, Rao RP, Anderson NR, Moran DW, Miller JW, Ojemann JG (2007d) Spectral changes in cortical surface potentials during motor movement. J Neurosci 27:2424-2432.

Murthy VN, Fetz EE (1996) Synchronization of neurons during local field potential oscillations in sensorimotor cortex of awake monkeys. J Neurophysiol 76:3968-3982.

Ojemann G, Ojemann J, Lettich E, Berger M (1989) Cortical language localization in left, dominant hemisphere. An electrical stimulation mapping investigation in 117 patients. J Neurosurg 71:316-326.

Penfield W, Boldrey E (1937) Somatic motor and sensory representation in the cerebral cortex of man as studied by electrical stimulation. Brain 37:389-443.

Pfurtscheller G (1999) Event-related desynchronization (ERD) and event related synchronization (ERS). Baltimore: Williams and Wilkins.

Pfurtscheller G, Graimann B, Huggins JE, Levine SP, Schuh LA(2003) Spatiotemporal patterns of beta desynchronization and gamma synchronization in corticographic data during self-paced movement. Clin Neurophysiol 114:1226-1236.

Phillips CG, Porter R (1977) Corticospinal neurones. Their role in movement. Monogr Physiol Soc 34:v-xii, 1-450.

Porter R, Lemon R (1993) Corticospinal function and voluntary movement. Oxford: Oxford UP.

Schalk G, McFarland DJ, Hinterberger T, Birbaumer N, Wolpaw JR (2004) BCI2000: a general-purpose brain-computer interface (BCI) system. IEEE Trans Biomed Eng 51:1034-1043.

Schieber MH (1995) Muscular production of individuated finger movements: the roles of extrinsic finger muscles. J Neurosci 15:284-297.

Schieber MH (1999) Somatotopic gradients in the distributed organization of the human primary motor cortex hand area: evidence from small infarcts. Exp Brain Res 128:139-148.

Schieber MH (2001) Constraints on somatotopic organization in the primary motor cortex. J Neurophysiol 86:2125-2143.

Schieber MH, Hibbard LS (1993) How somatotopic is the motor cortex hand area? Science 261:489-492.

Shinoda Y, Zarzecki P, Asanuma H (1979) Spinal branching of pyramidal tract neurons in the monkey. Exp Brain Res 34:59-72.

Woolsey CN, Erickson TC, Gilson WE (1979) Localization in somatic sensory and motor areas of human cerebral cortex as determined by direct recording of evoked potentials and electrical stimulation. J Neurosurg 51:476-506.

Woolsey CN, Settlage PH, Meyer DR, Sencer W, Pinto Hamuy T, Travis AM (1952) Patterns of localization in precentral and "supplementary" motor areas and their relation to the concept of a premotor area. Res Publ Assoc Res Nerv Ment Dis 30:238-264. 